International Journal of Clinical Dermatology \& Research (IJCDR)

ISSN: 2332-2977

\title{
A Review Of Erythroid Differentiation Regulator 1 (ERDR1) As A Therapeutic Target In Skin Inflammation
}

Review Article

Saad AlSogair

Elite Derma Care Clinic, Dr. Layla Al-Onaizi Polyclinic, Khobar, 31952, Saudi Arabia.

\section{Abstract}

Inflammatory skin diseases remain as one of the most common problems in dermatology. Skin inflammation is a complex process that starts with the introduction of a stimulus such as an allergen or an antigen that triggers the skin to produce inflammatory substances called cytokines and chemokines. At present, the approved drugs for treating inflammatory skin conditions are corticosteroids, yet these can have negative side effects on the skin. Erythroid Differentiation Regulator 1 (Erdr1) is a cytokine previously thought to be connected with hemoglobin synthesis only but was recently found to be involved in inflammation. This review examined the role of Erdr1 as a therapeutic target in the treatment of inflammatory skin disorders. By reviewing previous studies, it was evident that Erdr1 has pro-apoptotic activities; it is able to induce apoptosis in melanoma cells by the regulation of factors such as Bcl-2 and Bax. Treatment with recombinant Erdr1 (r Erdr1) was also able to inhibit rosacea by inhibiting blood vessel growth or angiogenesis and preventing further infiltration of inflammatory cells. There was also significant reduction of erythema, inflammatory cell infiltration, and microvessel density with vascular endothelial growth factor (VEGF). Altogether, these studies suggest that Erdr1 has a therapeutic effect on inflammatory skin diseases, particularly psoriasis, rosacea and other skin disorders.

Keywords: Erythroid Differentiation Regulator1; Erdr1; Inflammatory Skin Disorders; Inflammatory Skin Diseases; Inflammatory Skin; Skin Inflammation; Psoriasis; Rosacea; Melanoma; Skin Cancer; Skin Cancers.

\section{Introduction}

Inflammatory skin diseases remain as one of the most common problems in dermatology. They often present unpleasant symptoms such as rashes, itchiness, swelling, hives and redness. They may also be chronic, as in the case of psoriasis, atopic dermatitis and rosacea. Acute inflammation of the skin typically arises from exposure to ultraviolet radiation, allergens or chemical irritants in soap, shampoos or hair dyes. This usually disappears after one to two weeks without any damage to the underlying skin. On the other hand, chronic inflammation gives rise to a sustained cellmediated immune response in the skin that lasts for months to years with remissions and exacerbations.

Skin inflammation is a complex process that starts with the introduction of a stimulus such as an allergen or an antigen that triggers the skin to produce inflammatory substances called cytokines and chemokines. These substances then bind to receptors in the skin tissues to create vasodilation and the activation of nerve cells. They also trigger the migration of immune cells from the blood to the skin where these cells trigger the release of more inflammatory substances such as enzymes, cytokines and free radicals that further create more skin damage.

At present, the approved drugs for treating inflammatory skin conditions are corticosteroids. Indeed these drugs are effective for eczema, atopic dermatitis, seborrheic dermatitis and all other atopic skin conditions. However they have negative side effects on the skin such as a skin thinning effect, immunosuppression, an increase in blood glucose levels and abnormalities in the adrenal gland. Studies are currently being conducted on possible targets for therapeutic agents that can effectively treat inflammatory skin conditions. One of these targets is Erythroid Differentiation Regulator 1 (Erdr1), a cytokine previously thought to be connected with hemoglobin synthesis only but was recently found to be involved in inflammation. This review aims to examine the role of

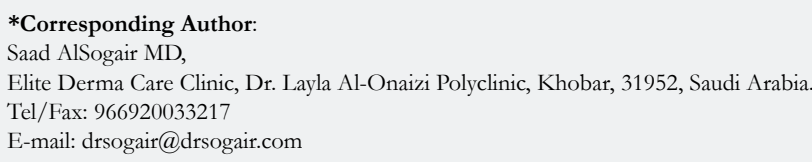

Copyright: Saad AlSogair ${ }^{\circ}$ 2020. This is an open-access article distributed under the terms of the Creative Commons Attribution License, which permits unrestricted use, distribution and reproduction in any medium, provided the original author and source are credited. 
Erdr1 as a therapeutic target in the treatment of inflammatory skin disorders.

\section{Methods}

A literature search was done on Pubmed using the search terms "erythroid differentiation regulator 1" and "erdr1". The articles were later scanned for relevant data. The bibliographies of the articles were further examined for relevant references. All the evaluations were done by the author. Relevant data from twelve (12) articles were included in this review.

\section{Results}

\section{Erythroid Differentiation Regulator 1}

Erythroid Differentiation Regulator 1 (Erdr1) is a cytokine which was initially known as a factor for hemoglobin synthesis in murine and human erythroleukemia cell lines [1]. This autocrine factor is found in normal cells and is situated in the inner portion of the cytoplasm, where it is secreted by the vesicles. Erdr1 is said to be secreted in response to stress and its action is necessary in the growth and survival of the hematopoietic system. Recent studies have shown that this factor is able to protect against cancer by limiting its growth and spread to other parts of the body. In one particular study [2], Erdr1 was able to limit the growth and spread of cancer cells by inducing E-cadherin and activating the immune system, particularly natural killer (NK) cells. Activated NK cells are then able to eliminate cancer cells by inducing apoptosis and cytolytic granule exocytosis.

Jung et al [2] reported that the expression of Erdr1 is negatively regulated by interleukin (IL)-18 which is a pro-inflammatory cytokine in murine cells. Increase in IL-18 is related to cancer progression in the form of increased proliferation, formation of new blood vessels (angiogenesis) and metastasis. Erdr1 has the opposite effect on cancer cells, as mentioned before.

IL-18 is also expressed in non-immune cells such as epithelial cells and keratinocytes. It also plays an essential role in skin inflammation. Thus, IL-18 suppression may be an effective therapeutic approach for inflammatory skin conditions. Recently, more research is being conducted with regards to the role of Erdr1 in treating and preventing inflammation because of its negative correlation with IL-18. This article aims to discuss the mechanism of action as well as the effects Erdr1 on inflammatory skin conditions, particularly psoriasis and rosacea.

\section{Mechanism of Action}

Erdr1 has pro-apoptotic activities; it is able to induce apoptosis in melanoma cells by the regulation of factors such as Bcl-2 and Bax [3]. It also acts by inhibiting blood vessel growth or angiogenesis and preventing further infiltration of inflammatory cells [4].

Under low cytokine levels, Erdr1 stimulated a seven-fold increase in the number of human haematopoietic progenitors [5]. These progenitors then gave rise to at least two lineages when they responded to Erdr1 and had more than one-fold increase in colony quantities. Erdr1 is released from irradiated stromal cells and can partly substitute for stromal cells in Burkitt's lymphoma, giving the impression that it functions as a stromal survival factor for stroma-responsive cells.

In mammalian cells, Erdr1 is mostly expressed as a $56 \mathrm{kDa}$ dimer and shows higher activity than the recombinant monomer [6]. The activity profile is bell-shaped. Expression was observed in many normal mouse tissues, yet in haematopoiesis it was largely confined to CD34+ cells. It was enhanced by a series of stimuli such as phorbol ester, and transformed cells generally showed a higher level of EDR expression than normal ones. The protein is localized at the inner side of the cytoplasmic membrane and is released in part via vesicles. In view of the broad range of EDRexpressing tissues the function obviously exceeds haemoglobin synthesis induction.

Erdr1 expression is present in primary keratinocytes and normal skin cells. It is enhanced by UVB radiation. PD98059, an extracellular signal-regulated kinase (ERK) inhibitor and SB203580, a mitogen-activated protein kinase (MAPK) inhibitor, greatly reduced Erdr1 expression after irradiation with UVB. Erdr1 is able to act through ERK and p38 MAPK pathways. There is a possibility that Erdr1 can be targeted by drugs to reduce apoptosis in keratinocytes as found in inflammatory skin conditions like arthritis and melanoma [7].

\section{Effects on the Skin}

Psoriasis: Psoriasis is a common skin disorder that is said to affect 2 to $3 \%$ of the global population. It presents as red and white scaly plaques in the topmost layer of the skin. Though the cause is not fully understood, research shows that immune-mediated inflammation is the culprit. Psoriasis may occur as a response to various stimuli such as infection, stress and allergens which, in turn, can stimulate keratinocytes to secrete cytokines and chemokines in the skin. This event then leads to the migration of immune cells such as neutrophils, dendritic cells, macrophages and Type 1 helper cells from the blood vessels to the skin. The skin cells, especially keratinocytes, proliferate at a fast rate and create the plaques for which psoriasis is known.

Some studies have proposed that interleukin-17 (IL-17) and tumor necrosis factor- $\alpha(\mathrm{TNF}-\alpha)$ are the cytokines responsible for keratinocyte activation and excessive proliferation, thus they can be used as markers for psoriasis. Other cytokines are also implicated in the pathogenesis of psoriasis. IL-18 can be recalled as a major cytokine for skin inflammation and is produced by keratinocytes, epithelial cells, monocytes and dendritic cells. The concentration of interleukin 18 in plasma is also related to psoriasis severity [4].

Kim et al., in 2016 studied the effects of Erdr1 on skin inflammation especially that in psoriatic skin triggered by imiquimod treatment. Recombinant Erdr1 significantly decreased skin inflammation in psoriasis and its accompanying signs and symptoms such as redness and scaling. It also decreased inflammatory cytokine levels especially those regulated by Th17 cells.

Recombinant Erdr1 also down-regulated CCL20 and CCR6, further giving the impression that Erdr1 relieves skin inflammation from psoriasis by regulating the presence of Th17 cell in affected skin. In summary, the study showed that Erdr1 may be useful in the treatment of psoriasis. 
Recombinant Erdr1 has also been shown to relieve inflammatory processes such as infiltration, thickening, skin desquamation, redness, scaling and itchiness. It also decreased the levels of biomarkers for psoriasis.

Interleukins 17 and 22 were also significantly decreased by rErdr1 in psoriatic skin. These cytokines are produced by Th17 cells and are the ones that trigger the hyperproliferation of the epidermis and skin barrier damage in psoriasis.

\section{Melanoma and Skin Cancers}

Melanoma is said to arise from chronic inflammation which in turn is triggered by ultraviolet (UV) radiation and other factors in the environment. Recently, studies have shown that inflammation in melanoma is due to the secretion of cytokines such as TNF, IL-1, IL-6, IL-18, matrix metalloproteinases (MMP), and vascular endothelial growth factor (VEGF). These cytokines are also responsible for other types of cancers.

Jung et al., [2] found that Erdr1 expression was higher in normal melanocytes compared with melanoma cell lines. This result was also confirmed in human skin from melanoma patients and healthy donors, indicating that Erdr1 expression is downregulated in melanoma. In the study, Erdr1 expression was found to be negatively regulated by IL-18, which has a pro-cancer effect on melanoma.

Overexpression of Erdr1 significantly inhibited cell migration, invasion, and proliferation in B16F10, a murine melanoma, in vitro. In addition, melanoma lung metastasis and tumor growth were suppressed in mice implanted with Erdr1-overexpressing melanoma. This study found that Erdr1 overexpression leads to the downregulation of heat shock protein 90 (HSP90), a ubiquitous chaperone that reportedly acts as a pro-cancer factor which is enhanced in advanced malignant melanoma related to stress conditions. Inhibition of HSP90 suggests that Erdr1 acts as a regulatory protein for melanoma motility.

Erdr1 was shown to decrease the growth of melanomas in mice by regulating apoptosis in vitro and in vivo. 8 Intraperitoneal injection of recombinant Erdr1 significantly reduced melanoma growth implanted onto mouse skin. Moreover, exogenous treatment of recombinant Erdr1 on B16F10 cells increased apoptosis by reducing $\mathrm{Bcl}-2$ expression while enhancing $\mathrm{Bax}$ in vitro.

In a study by Jung et al 9, it was found that Erdr1 overexpression markedly inhibited the level of cell migration, invasion, and proliferation in B16F10 cells in vitro. In addition, Erdr1 overexpression significantly suppressed melanoma lung colonization, metastasis, and tumor growth in vivo.

According to Lee et al., 10, Erdr1 is strongly expressed in the nuclei of normal skin cells, sebaceous gland, blood vessels, eccrine glands and nerves. In skin affected with seborrheic keratosis, eccrine spiradenoma and sebaceous hyperplasia, it was noted to be weak. It was rarely observed in malignant skin cancers such as malignant melanoma, squamous cell carcinoma and basal cell carcinoma.

\section{Rosacea}

Rosacea is another common inflammatory skin disorder that often affects the central tissues of the face, such as the nose, forehead, chin and cheeks. This condition is due to a complex interplay between blood vessels, nerves and immune cells in the skin. The proliferation of blood vessels or angiogenesis is one characteristic of rosacea and plays an important role in inflammation.

Studies have shown that the inflammation, vasodilation, fibrosis and neurosensory activation in rosacea are caused by the secretion of cytokines such as IL-18. Erdr1 expression has been examined in the skin tissues of patients with rosacea. Histological analysis revealed that Erdr1 expression is lower in patients with rosacea than normal controls. However, IL-18 exhibited a higher expression level in rosacea patients compared to healthy donors [11].

In addition, Erdr1 was able to treat rosacea in mice by regulation of angiogenesis [12]. Intraperitoneal administration of recombinant Erdr1 relieved clinical rosacea symptoms in a rosacea mouse model. Decreased VEGF expression and the suppression of CD34+ microvessel growth were also detected on the skin of Erdr1-treated animals, suggesting that Erdr1 suppresses angiogenesis. Additionally, an anti-inflammatory effect of Erdr1 mediated by reduced infiltration of inflammatory cells such as CD4+ and CD8+ T cells was demonstrated. These data suggest that Erdr1 suppresses T cell-mediated inflammation.

Treatment with recombinant Erdr1 (r Erdr1) was also able to inhibit rosacea by inhibiting blood vessel growth or angiogenesis and preventing further infiltration of inflammatory cells. Aside from the improvement of rosacea skin lesions, rErdr1 was shown to inhibit TNF- $\alpha$ production and chemokine secretion. These findings were also noted in psoriasis [4].

Kim et al [11]., were able to show that Erdr1 was downregulated, whereas IL-18 was upregulated, in patients with rosacea. Treatment with recombinant Erdr1 (rErdr1) resulted in a significant reduction of erythema, inflammatory cell infiltration, and microvessel density with vascular endothelial growth factor (VEGF).

\section{Conclusion}

This article aimed to review the effects of Erdr1 on inflammatory skin conditions. By reviewing previous studies, it was evident that Erdr1 has pro-apoptotic activities; it is able to induce apoptosis in melanoma cells by the regulation of factors such as Bcl-2 and Bax. Treatment with recombinant Erdr1 (r Erdr1) was also able to inhibit rosacea by inhibiting blood vessel growth or angiogenesis and preventing further infiltration of inflammatory cells. Inhibition of HSP90 suggests that Erdr1 acts as a regulatory protein for melanoma motility. Additionally, Erdr1 suppresses T cell-mediated inflammation. Treatment with recombinant Erdr1 (rErdr1) resulted in a significant reduction of erythema, inflammatory cell infiltration, and microvessel density with vascular endothelial growth factor (VEGF).

Altogether, these studies suggest that Erdr1 has a therapeutic effect on inflammatory skin diseases, particularly psoriasis, rosacea and other skin disorders. More studies are needed to ascertain 
whether Erdr1 has effects on other inflammatory skin conditions such as atopic dermatitis, alopecia and autoimmune disorders, as well as on skin cancers; safety studies in humans should also be done.

\section{References}

[1]. Houh YK, Kim KE, Park HJ, Cho D. Roles of Erythroid Differentiation Regulator 1 (Erdr1) on Inflammatory Skin Diseases. International Journal of Molecular Sciences. 2016; 17(12): 2059. Pubmed PMID: 27941650.

[2]. Jung MK, Houh YK, Ha S, Yang Y, Kim D, Kim TS, et al. Recombinant Erdr1 suppresses the migration and invasion ability of human gastric cancer cells, SNU-216, through the JNK pathway. Immunol. Lett. 2013; 150: $145-151$.

[3]. Lee J, Jung MK, Park HJ, Kim KE, Cho D. Erdr1 Suppresses Murine Melanoma Growth via Regulation of Apoptosis. International Journal of Molecular Sciences. 2016; 17(1): 107. Pubmed PMID: 26784177.

[4]. Kim KE, Kim S, Park S, Houh Y, Yang Y, Seung Beom Park, et al. Therapeutic effect of erythroid differentiation regulator 1 (Erdr1) on collagen-induced arthritis in DBA/1J mouse. Oncotarget. 2016 Nov 22; 7(47): 76354-76361. Pubmed PMID: 27823968

[5]. Dörmer P, Spitzer E, Möller W. EDR is a stress-related survival factor from stroma and other tissues acting on early haematopoietic progenitors (E-Mix). Cytokine. 2004; 27(2-3): 47-57. Pubmed PMID: 15242693.
[6]. Dörmer P, Spitzer E, Frankenberger M, Kremmer E. Erythroid differentiation regulator (EDR), a novel, highly conserved factor I. Induction of haemoglobin synthesis in erythroleukaemic cells. Cytokine. 2004; 26(6): 23142. Pubmed PMID: 15183840.

[7]. Kim HJ, Song SB, Yang Y, Eun YS, Cho BK, Hyun Jeong Park, et al. Erythroid differentiation regulator 1 (Erdr1) is a proapototic factor in human keratinocytes. Exp Dermatol. 2011 Nov; 20(11): 920-5. Pubmed PMID: 21995813.

[8]. Lee HR, Huh SY, Hur DY, Jeong H, Kim TS, Kim SY, et al. Erdr1 enhances human NK cell cytotoxicity through an actin-regulated degranulationdependent pathway. Cell. Immunol. 2014; 292: 78-84. Pubmed PMID: 25460082.

[9]. Jung MK, Park Y, Song SB, Cheon SY, Park S, Younkyung Houh, et al. Erythroid differentiation regulator 1 , an interleukin 18-regulated gene, acts as a metastasis suppressor in melanoma. J Invest Dermatol. 2011 Oct; 131(10): 2096-104. Pubmed PMID: 21697887.

[10]. Lee YB, Kim HJ, Jung HY, Park YG, Kim SY, Baik Kee Cho, et al. Downregulation of erythroid differentiation regulator 1 as a novel marker of skin tumors. Int J Dermatol. 2014 Jun; 53(6): 723-30. Pubmed PMID: 24168163.

[11]. Kim M, Kim KE, Jung HY, Jo H, Jeong SW, Lee J, et al. Recombinant erythroid differentiation regulator 1 inhibits both inflammation and angiogenesis in a mouse model of rosacea. Exp. Dermatol. 2015; 24: 680-685. Pubmed PMID: 25940661.

[12]. Park CC, Morel JC, Amin MA, Connors MA, Harlow LA, Koch AE. Evidence of IL-18 as a novel angiogenic mediator. J. Immunol. 2001; 167: 1644-1653. Pubmed PMID: 11466388. 